\title{
Assessment of Prognostic Factors and Adjuvant Treatment Modalities in Adult Head and Neck Soft Tissue Sarcoma Patients Treated With Upfront Surgery
}

Baran Akagündüz ${ }^{1}$, Tugba Akin Telli ${ }^{2}$, Sema Sezgin Goksu ${ }^{3}$, Hasan Cagri Yildirim ${ }^{4}$, Muhammet Ozer ${ }^{5}$, Sabin Göktaş Aydin ${ }^{6}$, Neslihan Ozyurt ${ }^{7}$, Cengiz Karacin ${ }^{8}$, Semra Paydas ${ }^{9}$, Mutlu Dogan 10

1. Medical Oncology, Erzincan Binali Yıldırım Üniversitesi Mengücek Gazi Hastanesi, Erzincan, TUR 2. Medical Oncology, Marmara University Medical School, Istanbul, TUR 3. Medical Oncology, Akdeniz University Medical School, Antalya, TUR 4. Medical Oncology, Hacettepe University Medical School, Ankara, TUR 5. Internal Medicine, Capital Health Regional Medical Center, Trenton, USA 6. Medical Oncology, Medipol Unıversity Medical School, Istanbul, TUR 7. Medical Oncology, Giresun Research Hospital, Giresun, TUR 8. Oncology, Recep Tayyip Erdoğan University Medical School, Rize, TUR 9. Medical Oncology, Cukurova University Faculty of Medicine, Adana, TUR 10. Medical Oncology, Ankara Dr Abdurrahman Yurtaslan Oncology Training and Research Hospital, Ankara, TUR

Corresponding author: Baran Akagündüz, drbaran04@hotmail.com

\section{Abstract \\ Objectives}

Head and neck soft tissue sarcomas (HNSTSs) are a heterogeneous group of rare tumors. Surgical resection with negative margins remains the standard primary treatment for patients with HNSTS. The role of chemotherapy (CT) and radiotherapy (RT) remains controversial. In this multicenter study, we aimed to demonstrate the real-world assessing prognostic factors and the effect of adjuvant treatment modalities in adult patients with HNSTS treated with upfront surgery.

\section{Methods}

We included a total of 47 patients who underwent curative-intent resection of a primary HNSTS between 2000 and 2019.

\section{Results}

The median follow-up was 29 months. The median age of patients was 51 years, and $66 \%$ of patients were male. The median relapse-free survival (RFS) of the study population was 31 months (range: 1.0-61.1 months), and the median overall survival (OS) was 115 months (range: 60.8-169.2 months). The univariable analysis revealed that treatment modalities showed a significant impact on RFS $(p=0.021)$; however, no difference was found in its impact on OS $(p=0.137)$. R0 resection did not showed impact on RFS $(p=0.130)$, but a significant association was found with OS $(\mathrm{p}=0.004)$. In multivariable analysis, $\mathrm{T}$ stage of the tumor (hazard ratio [HR]: $3.834 ; 95 \% \mathrm{CI}: 1.631-9.008 ; \mathrm{p}=0.002$ ) and treatment with surgery and sequential RT and CT (HR: 0.115 ; 95\% CI: 0.035-0.371; p < 0.001) were independent factors associated with RFS. R0 resection was independently associated with OS (HR: 4.902; 95\% CI: 1.301-18.465; p = 0.019).

\section{Conclusion}

Our study revealed that R0 resection improved OS, and T3-4 stage of tumor was a negative independent factor for RFS in surgically resected HNSTS patients. The use of sequential CT and RT after resection was associated with a better RFS, which emphasizes the importance of multidisciplinary evaluation of the treatment of HNSTS. Randomized prospective studies are needed

Categories: Radiation Oncology, Oncology

Keywords: adjuvant chemotherapy, survival analysis, head and neck sarcoma, adjuvant radiation therapy, head and neck cancer surgery

\section{Introduction}

Sarcomas are a rare and heterogeneous group of malignant neoplasms derived from mesenchymal tissues. They constitute approximately $1 \%$ of all malignancies in the head and neck region [1]. Surgery is the primary treatment of head and neck soft tissue sarcoma (HNSTS). Surgical removal of HNSTS cannot achieve the ideal "wide" resection margins due to their proximity to vital structures and the relatively small space of the head and neck region [2]. Sarcomas generally do not occur in the head and neck region; however, in patients with HNSTS, local recurrence mainly causes mortality [3]. For certain sarcomas, including rhabdomyosarcoma, Ewing sarcoma, and osteosarcoma, chemotherapy (CT) has emerged as a highly 
effective treatment modality [4-6]. The efficacy of the CT in the neoadjuvant or adjuvant setting for other histologies remains controversial [7-9]. Radiotherapy (RT) is an essential component of multimodality therapy, especially in treating patients with high grade or positive margins following surgical resection $[2,10,11]$. HNSTSs are rare diseases, and the current literature mainly consists of retrospective and small case series. This study aimed to determine the prognostic factors and the efficacy of postoperative adjuvant treatments in 47 HNSTS patients treated with upfront surgery.

\section{Materials And Methods}

\section{Study population}

This retrospective study included 47 patients diagnosed with HNSTS between 2010 and 2020 at 10 experienced medical oncology departments in Turkey. Patients who underwent curative-intent resection of a primary HNSTS without neoadjuvant therapy were included in the study. Exclusion criteria were aged $<18$ years, metastatic disease at diagnosis, treatment with neoadjuvant CT or RT for locally advanced disease, and patients with the diagnosis of Ewing's family sarcoma, alveolar or embryonal rhabdomyosarcoma, desmoid-type fibromatosis, and osteosarcoma. The patients receiving adjunctive RT with less than the radical dose ( $45 \mathrm{~Gy}$ ) were excluded. The wide excision and radical neck dissection were performed in all patients. The patients with lymph node involvement were excluded. The patients with missing data and those who had secondary primary cancer were also excluded.

\section{Data collection}

Data were retrieved from prospectively maintained databases in place at each participating institution. Clinical and demographic features including age, gender, histological subtype, pathological grade according to the FNCLCC (Fédération Nationale des Centres de Lutte Contre Le Cancer) grading system, surgical margin status, tumor size, stage according to the American Joint Committee on Cancer [AJCC] 8th Edition, necrosis, lymphovascular invasion, and the presence of adjuvant RT, CT, or both. Tumor margins were classified as complete (R0) or incomplete (R1/R2). Overall survival (OS) was defined as the time from the diagnosis to death or last follow-up. The relapse-free survival (RFS) was defined as the time from the diagnosis to the relapse or death.

\section{Ethical consideration}

This multicenter retrospective study was performed per the Declaration of Helsinki. It was reviewed and approved by the Ethics Committee of the University of Erzincan Binali Yıldırım University School of Medicine.

\section{Statistical analysis}

IBM SPSS 25 (Statistics Program for Social Scientists, IBM Corp., Armonk, NY, USA) was used for statistical analysis. Kolmogorov-Smirnov test was used for the compatibility of the data to normal distribution. Nonparametric continuous data were presented as median (range), and categorical data were presented as frequency (percentage). Survival analysis was performed using the Kaplan-Meier method. Log-rank test was used to compare survival times between groups. The independent prognostic factors for OS and RFS were determined using the Cox regression analysis. The time from diagnosis to death due to any reason was defined as OS, and the time from diagnosis to disease relapse or death was defined as RFS. All statistical tests were performed bilaterally, and $\mathrm{p}<0.05$ was considered statistically significant.

\section{Results}

\section{Clinicopathological features}

A total of 47 patients diagnosed with HNSTS between 2010 and 2019 were included in this study. The median follow-up was 29 months (range: 8-163 months). Patients' characteristics are described in Table 1. Of the patients, 16 (34\%) were female and 31 (66\%) were male. The median age of patients was 51 years (range: $18-85$ years). Of the patients, $42.5 \%$ had grade 3 disease, $29.8 \%$ had grade 2 disease, and $27.7 \%$ had grade 1 disease. Also, $14.9 \%$ of patients had stage T1 disease, $27.7 \%$ had stage T2 disease, 51.1\% had stage T3 diseases, and $6.3 \%$ had stage T4 disease. Necrosis was found in $17 \%$ of the patients, and vascular invasion was found in $55.3 \%$ of patients. In our cohort, $25.2 \%$ of patients had synovial sarcoma, $12.6 \%$ had leiomyosarcoma, $12.6 \%$ had myxofibrosarcoma, $12.6 \%$ spindle cell sarcoma, $8.4 \%$ angiosarcoma, $6.3 \%$ had undifferentiated pleomorphic sarcoma, and $21 \%$ of patients had other histological types. Also, $50.3 \%$ of patients had neck sarcoma, $19.1 \%$ had scalp/face sarcoma, $17 \%$ had supraclavicular sarcoma, and $10.7 \%$ had paranasal sinus sarcoma.

$$
n=47
$$




\section{Cureus}

\section{Male}

$31(66.0)$

Female

16 (34.0)

Tumor grade, $\mathrm{n}$ (\%)

1

13 (27.7)

2

14 (29.8)

3

20 (42.5)

Necrosis

Yes

8 (17.0)

No

39 (83.0)

LVI

Yes

$26(55.3)$

No

21 (44.7)

T stage, n (\%)

T1

7 (14.9)

T2

13 (27.7)

T3

24 (51.1)

T4

$3(6.3)$

R0 resection, $\mathrm{n}(\%)$

Yes

36 (76.6)

No

11 (23.4)

Treatment modality, $\mathrm{n}(\%)$

Surgery

14 (29.8)

Surgery + RT

6 (12.8)

Surgery + CT

10 (21.3)

Surgery + RT + CT

17 (36.1)

Histological subtypes, n (\%)

Synovial sarcoma

12 (25.2)

Leiomyosarcoma

6 (12.6)

Myxofibrosarcoma

$6(12.6)$

Spindle cell sarcoma

6 (12.6)

Angiosarcoma

4 (8.4)

Undifferentiated pleomorphic sarcoma

$3(6.3)$

Others

$10(21.0)$

Tumor location

Neck

Scalp/face

Supraclavicular

8 (17.0)

Paranasal sinus

5 (10.7)

Chemotherapy regimen 


\section{Cureus}

IMA

$24(51.1)$

Paclitaxel

$1(2.1)$

Ifosfamide

$1(2.1)$

Adriamycin

None

$20(42.6)$

\section{TABLE 1: Patients' and treatment characteristics}

CT, chemotherapy; IMA, ifosfamide and adriamycin; LVI, lymphovascular ınvasion; OS, overall survival; RFS, relapse-free survival; RT, radiotherapy

\section{Treatment}

In regard to treatment, $29.8 \%$ of patients were treated with surgery alone, $12.8 \%$ with surgery and adjuvant RT, $21.3 \%$ with surgery and adjuvant CT, and $36.1 \%$ with surgery and sequential CT and RT. Also, $51.1 \%$ of patients were treated with ifosfamide and adriamycin, $2.1 \%$ with ifosfamide, $2.1 \%$ with adriamycin, and $2.1 \%$ with paclitaxel.

\section{Survival analysis}

The median RFS of the study population was 31 months (range: 1.0-61.1 months), and the median OS was 115 months (range: 60.8-169.2 months). In univariable analysis, treatment modality showed a significant impact on RFS ( $(\mathrm{p}=0.021)$; however, no significant association was present with OS $(\mathrm{p}=0.137)$ (Figures 1, 2). The T stage of the tumor did not affect RFS $(p=0.320)$ but affected OS $(p<0.001)$ (Figures 3, 4). R0 resection did not showed impact on RFS ( $p=0.130)$ but showed a significant association with OS $(\mathrm{p}=0.004)$ (Figures 5 , ๑). Tumor grade, gender, necrosis, and lymphovascular invasion did not affect OS and RFS (Table 2).

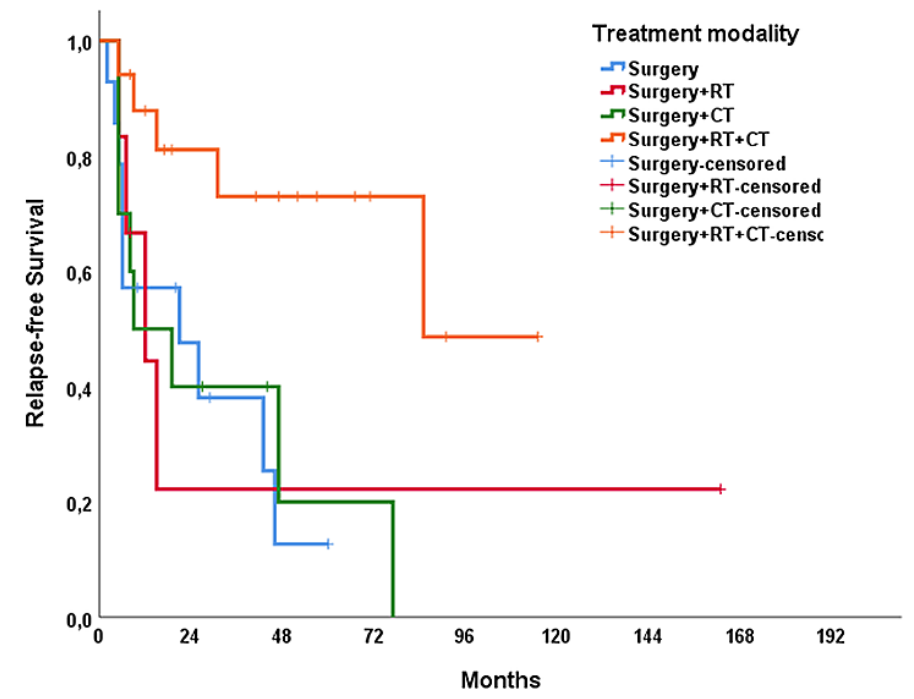

FIGURE 1: Relapse-free survival by treatment modalities 


\section{Cureus}

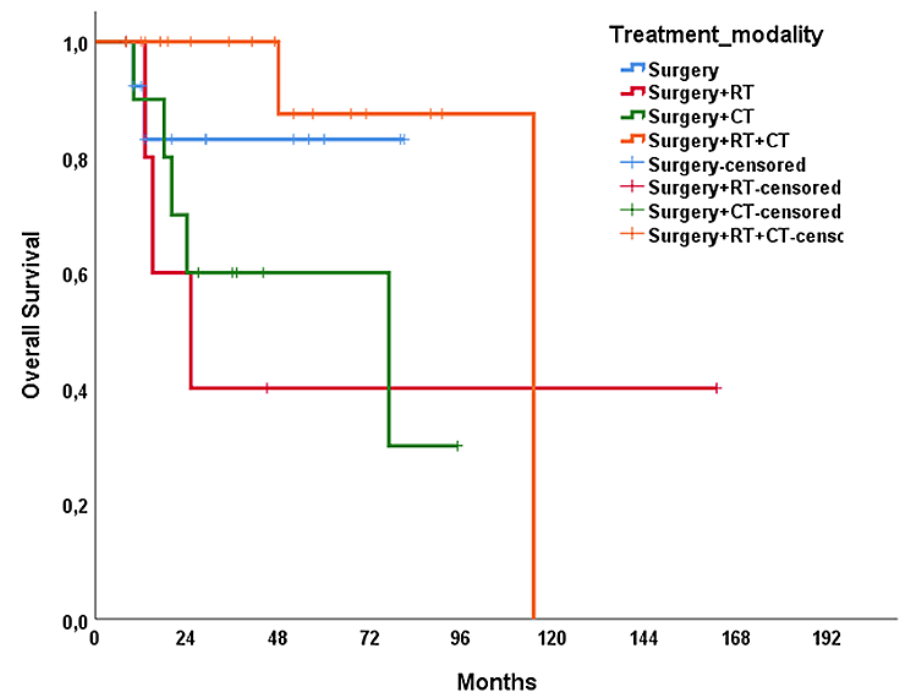

FIGURE 2: Overall survival by treatment modalities

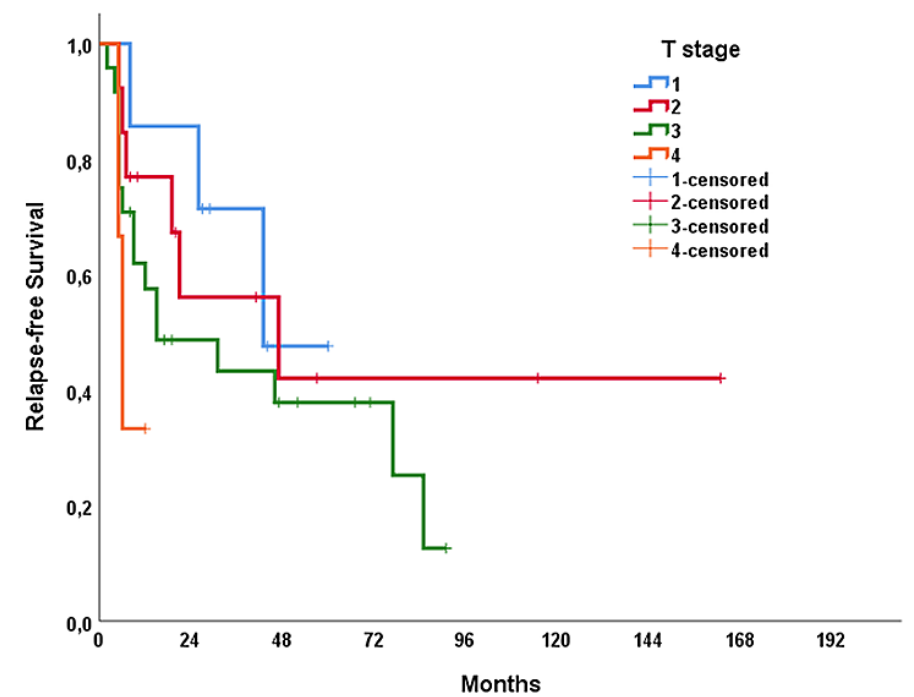

FIGURE 3: Relapse-free survival by T stage

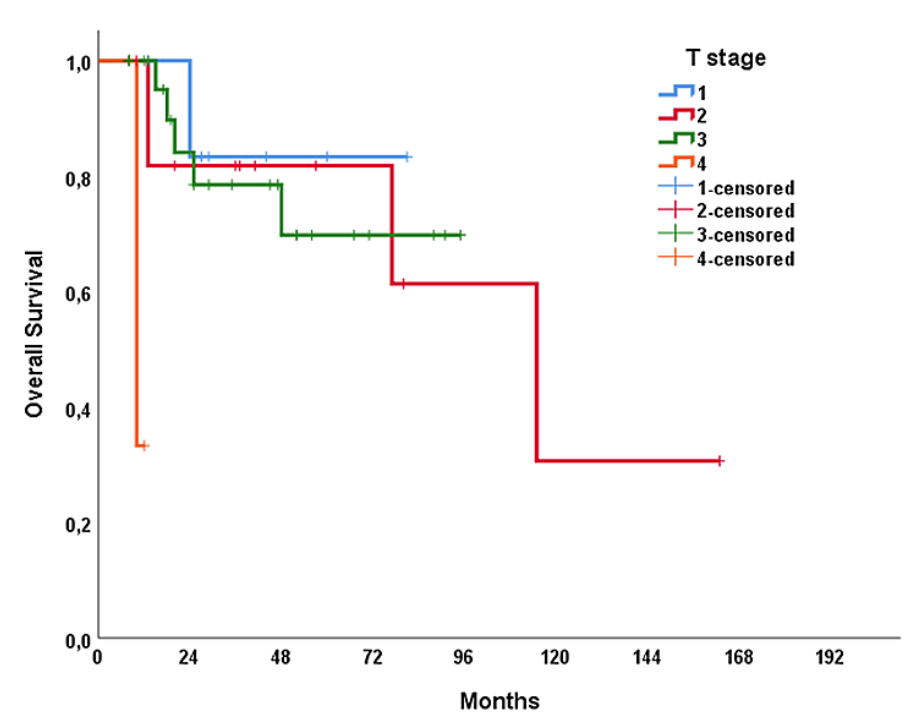




\section{Cureus}

FIGURE 4: Overall survival by T stage

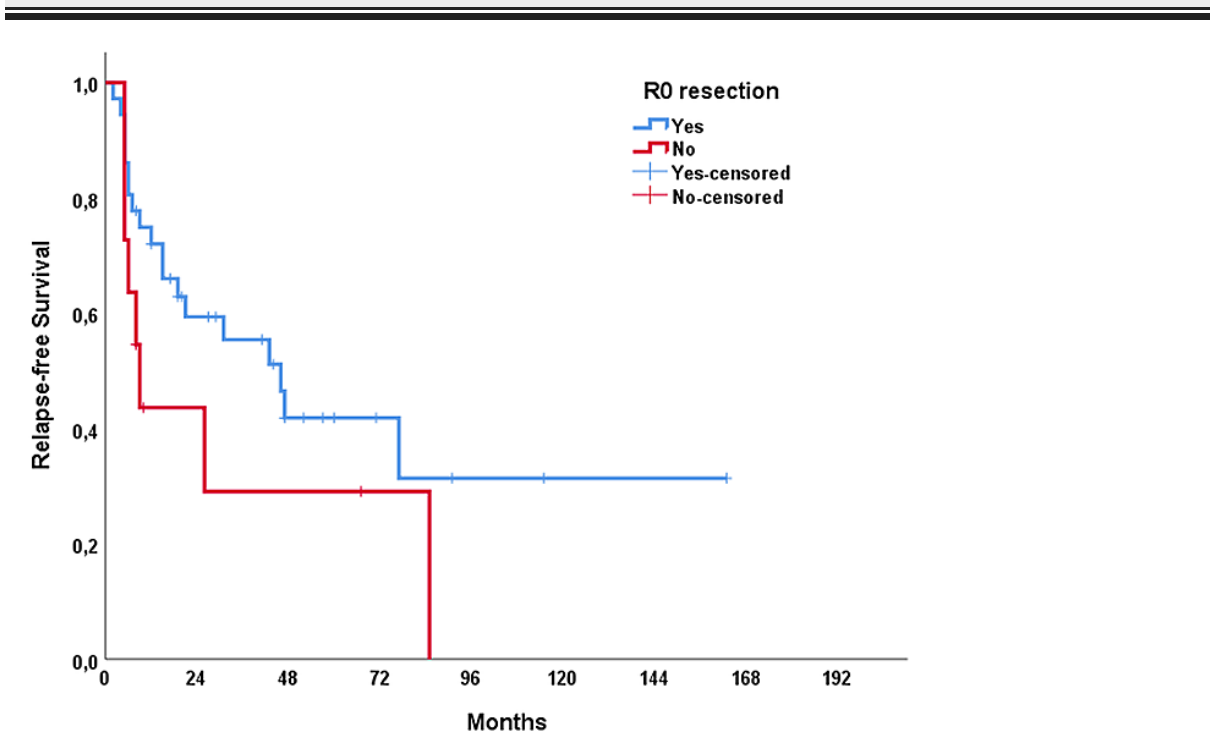

FIGURE 5: Relapse-free survival by $\mathrm{R0}$ resection

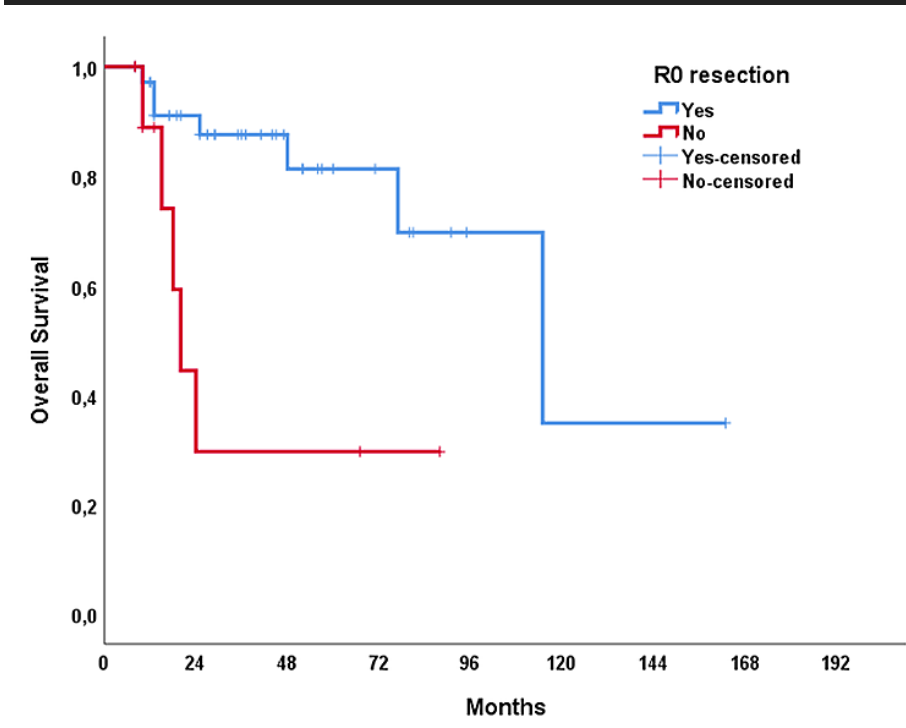

FIGURE 6: Overall survival by R0 resection 


\section{Cureus}

\begin{tabular}{|c|c|c|c|c|}
\hline & RFS, months (95\% Cl) & p-Value & OS, months (95\% Cl) & p-Value \\
\hline All patients & $31(1.0-61.1)$ & - & $115(60.8-169.2)$ & - \\
\hline \multicolumn{5}{|l|}{ Gender } \\
\hline Male & $43(7.9-78.0)$ & 0.579 & 77 (24.1-129.9) & 0.235 \\
\hline Female & $31(1.0-67.2)$ & & NR & \\
\hline \multicolumn{5}{|l|}{ Tumor grade } \\
\hline $1-2$ & $43(17.5-68.5)$ & 0.683 & NR & 0.089 \\
\hline 3 & $15(1.0-56.5)$ & & $77(1.0-153.0)$ & \\
\hline \multicolumn{5}{|l|}{ Necrosis } \\
\hline Yes & $31(0-76.1)$ & 0.928 & 115 (NA) & 0.658 \\
\hline No & $26(0-55.9)$ & & NR & \\
\hline \multicolumn{5}{|l|}{ LVI } \\
\hline Yes & $31(2.9-59.1)$ & 0.356 & $115(19.0-210.9)$ & 0.417 \\
\hline No & $77(0-217.8)$ & & NR & \\
\hline \multicolumn{5}{|l|}{ Tumor stage } \\
\hline T1 & 43 (NA) & 0.320 & NR & $<0.001$ \\
\hline T2 & $47(1.0-107.7)$ & & 115 (57.1-172.9) & \\
\hline T3 & $15(1.0-42.1)$ & & NR & \\
\hline T4 & $6(4.4-7.6)$ & & 10 (NA) & \\
\hline \multicolumn{5}{|l|}{ R0 resection } \\
\hline Yes & $46(24.3-67.7)$ & 0.130 & $115(60.6-169.4)$ & 0.004 \\
\hline No & $9(4.4-13.5)$ & & $20(15.0-24.9)$ & \\
\hline \multicolumn{5}{|l|}{ Treatment modality } \\
\hline Surgery & $21(0-49.9)$ & 0.021 & NR & 0.137 \\
\hline Surgery + RT & $12(2.2-21.8)$ & & $25(3.5-46.4)$ & \\
\hline Surgery + CT & $9(0-26.0)$ & & $77(0-155.2)$ & \\
\hline Surgery + RT + CT & 85 (NA) & & 115 (NA) & \\
\hline \multicolumn{5}{|l|}{ Tumor location } \\
\hline Neck & $26(0-62.3)$ & 0.924 & $115(19.3-210.7)$ & 0.997 \\
\hline Scalp/tace & $19(0-43.4)$ & & NR & \\
\hline Supraclavicular & $47(0-110.7)$ & & 77 (NA) & \\
\hline Paranasal sinus & $31(0-68.8)$ & & NR & \\
\hline
\end{tabular}

\section{TABLE 2: Univariable analysis of factors affecting OS and RFS}

CT, chemotherapy; LVI, lymphovascular Invasion; NA, not applicable; NR, not reached; OS, overall survival; RFS, relapse-free survival; RT, radiotherapy

Multivariable analysis revealed that $\mathrm{T}$ stage of the tumor (hazard ratio [HR]: $3.834 ; 95 \% \mathrm{CI}: 1.631-9.008 ; \mathrm{p}=$ 0.002), and treatment with surgery and sequential RT and CT (HR: 0.115; 95\% CI: 0.035-0.371; p 0.001) were independent factors associated with RFS. Only R0 resection (HR: 4.902; 95\% CI: 1.301-18.465; $\mathrm{p}=$ 0.019) was independently associated OS (Table 3). 


\section{Cureus}

\begin{tabular}{|c|c|c|c|c|}
\hline & \multicolumn{2}{|l|}{ RFS } & \multicolumn{2}{|l|}{ OS } \\
\hline & HR (95\% Cl) & $p$-Value & HR (95\% Cl) & $\mathrm{p}$-Value \\
\hline \multicolumn{5}{|l|}{ T stage } \\
\hline T1-2 & Reference & 0.002 & Reference & 0.204 \\
\hline T3-4 & $3.834(1.631-9.008)$ & & $2.392(0.623-9.181)$ & \\
\hline \multicolumn{5}{|l|}{ Tumor grade } \\
\hline $1-2$ & - & - & Reference & 0.086 \\
\hline 3 & - & & 2.945 (0.859-10.099) & \\
\hline \multicolumn{5}{|l|}{ R0 resection } \\
\hline Yes & Reference & 0.203 & Reference & 0.019 \\
\hline No & $1.795(0.730-4.416)$ & & 4.902 (1.301-18.465) & \\
\hline \multicolumn{5}{|l|}{ Treatment modality } \\
\hline Surgery & Reference & & - & - \\
\hline Surgery + RT & $0.675(0.204-2.235)$ & 0.520 & - & \\
\hline Surgery + CT & $0.801(0.307-2.088)$ & 0.650 & - & - \\
\hline Surgery + RT + CT & $0.115(0.035-0.371)$ & $<0.001$ & - & - \\
\hline
\end{tabular}

\section{TABLE 3: Multivariable analysis of factors affecting OS and RFS}

$\mathrm{CT}$, chemotherapy; RT, radiotherapy; RFS, relapse-free survival; OS, overall survival

\section{Discussion}

Soft tissue sarcomas account for about $1 \%$ of all malignancies in the head and neck region and $4-10 \%$ of all adult sarcomas $[12,13]$. The primary goal of surgical tumor resection should be obtaining local control with negative margins at the first attempt, preventing increased morbidity, and having the best chance for a possible cure. Around 30\% of HNSTSs occur in children. The median age at diagnosis is between 50 and 54 years in all patients, and when pediatric patients are not included, the median age at diagnosis is between 55 and 59 years [14]. The median age in the study population was 51 years (range: 18 -85 years). There was a male predominance (66\%) in our cohort, similar to the previous series [15]. In our study, the most common histological subtype was synovial sarcoma, whereas we excluded osteogenic sarcomas, non-chemosensitive and non-radiosensitive sarcomas consisting of alveolar soft part sarcomas, dermatofibrosarcoma protuberans, and gastrointestinal stromal tumors in this study. We found that the T3-4 tumor stage was an independent predictor of poor RFS in the multivariable analysis but did not affect OS. In an Italian retrospective cohort of 101 patients, it was demonstrated that AJCC 8 T classification cut-off points were not significantly different on multivariable analysis [16]. Another recent study revealed that the currently used AJCC 8 T classification cut-off points were not prognostic [17]. Positive surgical margins are associated with poor prognosis [18]. In a series of 146 patients with a variety of skull base sarcomas (both of soft tissue and bone), five-year disease-specific survival rates were $77 \%$, $43 \%$, and $36 \%$ for those with negative, close (often defined as $<1 \mathrm{~mm}$ ), and positive surgical margins, respectively, and the presence of positive or close margins was the only independent predictor of poor survival in multivariable analysis [19]. Our study showed that R0 resection was a positive predictor factor for OS but did not relate to RFS. In one study of 122 sarcomas of the head or neck, patients with high-grade lesions had significantly worse survival compared with those with low-grade lesions (HR for death: 5.52; 95\% CI: 1.51-20.21) [20]. In our study, no correlation was found between tumor grade and OS and RFS. Also, we found that necrosis, lymphovascular invasion, and gender had no effect on OS and RFS. The low number of cases could explain these results.

Recommendations for adjuvant CT and/or RT are made for each case based on multidisciplinary evaluation of all clinical and histopathological features, tumor CT and radiation sensitivity, margin status, and highrisk conditions. Park et al. demonstrated that RT and CT were not associated with improved disease-specific survival or OS [20]. Mattavelli et al. showed that adjuvant RT did not demonstrate a survival benefit [21]. In a recent study, Mahmoud et al. demonstrated that adjuvant RT was associated with improved survival in highgrade HNSTS [22]. Most of our study population received adjuvant RT and CT sequentially. In the multivariable analysis, we showed that adjuvant RT and CT improved RFS. In univariable analysis, treatment 
modality did not affect OS. Adjuvant only CT or only RT did not demonstrate any survival benefit.

Our study had some limitations. First of all, it is a retrospective analysis of patients from various medical oncology departments. Histopathological evaluations of the patients may vary depending on the experience of institutions. Unfortunately, we could not have had a pathological reevaluation of the paraffin blocks by the same pathologist. Therefore, the lack of a central pathological assessment is another limitation of this study. Our study consisted of a small sample size. We could not have had any molecular evaluation in our patients. On the other hand, our study offers real-life data.

\section{Conclusions}

Our study revealed that R0 resection improved OS, and T3-4 stage of tumor was a negative independent factor for RFS in surgically resected HNSTS patients. The use of sequential CT and RT after resection was associated with a better RFS, which emphasized the importance of multidisciplinary evaluation of the treatment of HNSTS. Randomized prospective studies are needed to determine the adjuvant treatment strategies and prognostic factors of HNSTS. Identification of possible biomarkers may enable us to tailor indications and choice of adjuvant treatment modality in patients with HNSTS.

\section{Additional Information}

\section{Disclosures}

Human subjects: Consent was obtained or waived by all participants in this study. University of Erzincan Binali Yıldırım University School of Medicine issued approval 33216249-50.01.02. Animal subjects: All authors have confirmed that this study did not involve animal subjects or tissue. Conflicts of interest: In compliance with the ICMJE uniform disclosure form, all authors declare the following: Payment/services info: All authors have declared that no financial support was received from any organization for the submitted work. Financial relationships: All authors have declared that they have no financial relationships at present or within the previous three years with any organizations that might have an interest in the submitted work. Other relationships: All authors have declared that there are no other relationships or activities that could appear to have influenced the submitted work.

\section{References}

1. Tudor-Green B, Fonseca FP, Gomez RS, Brennan PA: Current update on the diagnosis and management of head and neck hard tissue sarcomas. J Oral Pathol Med. 2017, 46:667-673. 10.1111/jop.12573

2. Sturgis EM, Potter BO: Sarcomas of the head and neck region . Curr Opin Oncol. 2003, 15:239-252. 10.1097/00001622-200305000-00011

3. Eeles RA, Fisher C, A'Hern RP, et al.: Head and neck sarcomas: prognostic factors and implications for treatment. Br J Cancer. 1993, 68:201-207. 10.1038/bjc.1993.314

4. Grier HE, Krailo MD, Tarbell NJ, et al.: Addition of ifosfamide and etoposide to standard chemotherapy for Ewing’s sarcoma and primitive neuroectodermal tumor of bone. N Engl J Med. 2003, 348:694-701. 10.1056/NEJMoa020890

5. Wolden SL, Anderson JR, Crist WM, et al.: Indications for radiotherapy and chemotherapy after complete resection in rhabdomyosarcoma: A report from the Intergroup Rhabdomyosarcoma Studies I to III. J Clin Oncol. 1999, 17:3468-3475. 10.1200/JCO.1999.17.11.3468

6. Rosen G, Caparros B, Nirenberg A, et al.: Ewing's sarcoma: ten-year experience with adjuvant chemotherapy. Cancer. 1981, 47:2204-2213. 10.1002/1097-0142(19810501)47:9<2204::AIDCNCR2820470916>3.0.CO;2-A

7. Pervaiz N, Colterjohn N, Farrokhyar F, Tozer R, Figueredo A, Ghert M: A systematic meta-analysis of randomized controlled trials of adjuvant chemotherapy for localized resectable soft-tissue sarcoma. Cancer. 2008, 113:573-581. 10.1002/cncr.23592

8. Glenn J, Kinsella T, Glatstein E, et al.: A randomized, prospective trial of adjuvant chemotherapy in adults with soft tissue sarcomas of the head and neck, breast, and trunk. Cancer. 1985, 55:1206-1214. 10.1002/1097-0142(19850315)55:6<1206::aid-cncr2820550612>3.0.co;2-e

9. Gortzak E, Azzarelli A, Buesa J, et al.: A randomised phase II study on neo-adjuvant chemotherapy for 'highrisk’ adult soft-tissue sarcoma. Eur J Cancer. 2001, 37:1096-1103. 10.1016/s0959-8049(01)00083-1

10. Pezzi CM, Rawlings MS Jr, Esgro JJ, Pollock RE, Romsdahl MM: Prognostic factors in 227 patients with malignant fibrous histiocytoma. Cancer. 1992, 69:2098-2103. 10.1002/1097-0142(19920415)69:8<2098::aidcncr2820690815>3.0.c0;2-9

11. Weber RS, Benjamin RS, Peters LJ, Ro JY, Achon O, Goepfert H: Soft tissue sarcomas of the head and neck in adolescents and adults. Am J Surg. 1986, 152:386-392. 10.1016/0002-9610(86)90309-0

12. Hoffman HT, Robinson RA, Spiess JL, Buatti J: Update in management of head and neck sarcoma . Curr Opin Oncol. 2004, 16:333-341. 10.1097/01.cco.0000127880.69877.75

13. Kraus DH, Dubner S, Harrison LB, et al.: Prognostic factors for recurrence and survival in head and neck soft tissue sarcomas. Cancer. 1994, 74:697-702. 10.1002/1097-0142(19940715)74:2<697::aidcncr2820740224>3.0.c0;2-a

14. Peng KA, Grogan T, Wang MB: Head and neck sarcomas: analysis of the SEER database . Otolaryngol Head Neck Surg. 2014, 151:627-633. 10.1177/0194599814545747

15. Freedman AM, Reiman HM, Woods JE: Soft-tissue sarcomas of the head and neck . Am J Surg. 1989, 158:367372. 10.1016/0002-9610(89)90135-9

16. Giannini L, Bresciani L, Paderno A, Incandela F, Fiore M, Gronchi A, Piazza C: Head and neck adult-type soft 


\section{Cureus}

tissues sarcomas: survival analysis and comparison between the last two editions of the TNM staging system [Online ahead of print]. Eur Arch Otorhinolaryngol. 2020, 10.1007/s00405-020-06452-3

17. Lee NCJ, Eskander A, Miccio JA, et al.: Evaluation of head and neck soft tissue sarcomapathologic staging system and proposal of a novel stage grouping system. Oral Oncol. 2021, 114:105137.

10.1016/j.oraloncology.2020.105137

18. Tran LM, Mark R, Meier R, Calcaterra TC, Parker RG: Sarcomas of the head and neck. Prognostic factors and treatment strategies. Cancer. 1992, 70:169-177. 10.1002/1097-0142(19920701)70:1<169::aidcncr2820700127>3.0.co; $-\mathrm{f}$

19. Gil Z, Patel SG, Singh B, et al.: Analysis of prognostic factors in 146 patients with anterior skull base sarcoma: an international collaborative study. Cancer. 2007, 110:1033-1041. 10.1002/cncr.22882

20. Park JT, Roh JL, Kim SO, Cho KJ, Choi SH, Nam SY, Kim SY: Prognostic factors and oncological outcomes of 122 head and neck soft tissue sarcoma patients treated at a single institution. Ann Surg Oncol. 2015, 22:248255. 10.1245/s10434-014-3870-8

21. Mattavelli D, Miceli R, Radaelli S, et al.: Head and neck soft tissue sarcomas: prognostic factors and outcome in a series of patients treated at a single institution. Ann Oncol. 2013, 24:2181-2189.

10.1093/annonc/mdt126

22. Mahmoud O, Beck R, Kalyoussef E, Chan Park R, Baredes S, Kim S, Samuels MA: Adjuvant therapies utilization pattern and survival outcomes in high-grade head and neck soft tissue sarcoma; a population based study. Oral Oncol. 2017, 66:28-37. 10.1016/j.oraloncology.2016.12.030 